\title{
Practical Definition Method of Laser Ablation Threshold in Nano-second Order Pulse Duration Considering Intensity Distribution at Focal Point
}

\author{
Haruhisa SAKAMOTO* $^{*}$ and Koji MORIOKA ${ }^{* *}$ \\ * Sophia University, Faculty of Science Technology, 7-1 Kioi-cho, Chiyoda-ku, \\ Tokyo 102-8554, Japan \\ E-mail:h-sakamo@sophia.ac.jp \\ ${ }^{* *}$ Sony Corporation, 1-7-1, Konan, Minato-ku, Tokyo, Japan
}

\begin{abstract}
The threshold of laser ablation means the minimum laser intensity that enables to remove material. This value should be inherent to the material but independent of the optical system configuration essentially. In this study, the novel definition of the threshold is proposed, and then, the values for various materials are experimentally determined to evaluate its validity. In the experiments, the pulsed YAG laser with the pulse width of $8 \mathrm{~ns}$ and the wavelength of $355 \mathrm{~nm}$ is scanned on silicone, aluminum and silver for groove machining. The ablation threshold can be determined as the power density corresponding to the groove edge. In case of silicone, the values of threshold are always $80 \mathrm{MW} / \mathrm{cm}^{2}$ independent of the laser intensity and its distribution. Furthermore, since the change in the focal length of the objective lens does not affect to the threshold determination, it is confirmed that the threshold is also independent of the optical system configuration. In case of aluminum and silver, the values of threshold are 135 and $40 \mathrm{MW} / \mathrm{cm}^{2}$ respectively, and are also independent of the laser fluence and the optical system configuration. The proposed definition enables estimation the ablation dimensions corresponding to the laser intensity and material independent of the optical system configuration.
\end{abstract}

DOI:10.2961/jlmn.2013.01.0010

Keywords: laser ablation, threshold power density, pulsed YAG laser, groove machining, intensity distribution, machined width, focal length

\section{Introduction}

Laser ablation is applied for various industrial purposes, such as removal process, such as micro boring [1], microcircuit repair [2] and evaporation process that includes thin film deposition [3] and ultra-small particle generation [4]. Recently, the laser sources are actively improved such as widening the wavelength range [5], extremely shortening pulse duration [6], enhancing pulse energy [7] and so on. Consequently, the expectation for laser ablation, which uses the improved sources, has risen more and more [8].

The laser ablation is the process that the irradiation of laser pulses of more than specific intensity, which is called threshold, breaks the bonds between atoms or molecules to remove the material from the solid surface minutely [9]. This process is affected by not only the workpiece material properties such as composition, absorption coefficient, and thermal conductivity, but also the laser irradiation characteristics such as wavelength, pulse duration, intensity and spot diameter. Therefore, for the process control in ablation, the laser irradiation conditions should be set appropriately corresponding to the workpiece material.

As mentioned above, the possibility of ablation depends upon whether the irradiated laser intensity exceeds threshold. Therefore, to determine the laser irradiation conditions, the grasp of threshold is essentially important.

The values of threshold have been examined experimentally for various materials. For instance, the ablation threshold values for the most of materials by nano-second pulse laser are reported as fluence of $0.1 \sim 10 \mathrm{~J} / \mathrm{cm}^{2}[10-14]$.
Like those, the ablation threshold had been generally determined by the average fluence, but neglecting the laser intensity distribution at the irradiation spot.

However, it is well known that the thresholds strongly depend upon not only the parameter peculiar to the laser oscillator such as wavelength and pulse duration, but also the parameter affected by the optical system configuration such as spot diameter and intensity distribution. However, since the detailed examination had been done not enough about those influences, the determination of the ablation condition is stayed in the empirical method, that is, it is not enough rational.

By physical thinking, the ablation threshold corresponds to the critical energy value for decomposition or evaporation of the workpiece material. Therefore, the value of threshold should be determined as the inherent value of each material essentially. In case of ultrafast pulsed laser ablation, it is reported that the ablation threshold can be determined corresponding to the irradiation intensity distribution [15]. However, it has not been clear whether the situation is same in case of laser ablation with nano-second order pulse duration.

In this study, for the laser ablation with nano-second order pulse duration, the practical definition is proposed to determine the ablation threshold as the inherent value for materials independent of the optical system configuration and the intensity distribution of irradiated laser. Furthermore, the validity of the method is examined experimental- 
ly with application to various materials and optical conditions.

\section{Experimental apparatus and methods}

In the experiment, the laser beam is delivered with some reflection mirrors, and focused on the flat specimen with three objective lenses different in the focal length. The irradiation intensity is controlled with the attenuator by polarization.

Table 1 shows the specification of the laser oscillator. Of the irradiated laser, the wavelength is $355 \mathrm{~nm}$, that is, the third harmonic of YAG laser, and the pulse duration is shortened less than $10 \mathrm{~ns}$ by using EO-Q switch. The oscillator generates the average power of $660 \mathrm{~mW}$ with pulse repetition rate of $500 \mathrm{~Hz}$, consequently, the pulse energy reaches $1.32 \mathrm{~mJ}$.

Figure1 shows the typical measurement result of beam mode of the irradiated laser. As shown in the figure, the mode is classified as the single-mode, and the correlation coefficient to ideal TEM $_{00}$ mode is 0.98 .

Figure 2 shows the schematic diagrams of laser irradiation method for groove machining. As shown in (a) in the figure, the grooves for evaluation can be obtained by moving the workpiece table in order to reciprocate the converged laser spot in one direction with the constant speed. At this time, the laser pulses are irradiated with the constant power and repetition rate. Furthermore, as shown in (b) in the figure, the table speed is set to make the spacing of the pulses equal to a half of $1 / \mathrm{e}^{2}$ spot diameter.

Table 2 shows the experimental conditions for groove machining. In order to evaluate the effect of the optical system configuration, the focal length $F$ of the objective lens is changed from 50 to $140 \mathrm{~mm}$. In addition, to evaluate the effect of the workpiece material, the materials selects silicon ( $\mathrm{Si})$, aluminum $(\mathrm{Al})$ and silver $(\mathrm{Ag})$.

Figure 3 shows the typical cross-sectional view of the machined groove and the definition of the machined width $W m$. By reciprocally irradiating laser pulses along a same line, the cross-section of the groove becomes a vee-shape finally. As shown in the figure, the machined width can be determined from the entrance width of a vee-shape.

\section{Novel definition of laser ablation threshold}

\subsection{Formulation of intensity distribution at focal point}

Figure 4 indicates the principle model of laser beam delivery in order to derive the intensity distribution of an irradiated laser at the focal point. (a) of the figure indicates the intensity distribution model of a $\mathrm{TEM}_{00}$ mode laser at the focal point.

Equation (1) expresses the power density $I(r)$ at radius $r$ from the beam center, here, $r_{0}$ means the representative radius for the Gaussian distribution and $a$ means the scale constant corresponding to the spot radius. Since the sum total of power $\triangle P$ within a doughnut-shape range of an infinitely small width $\Delta r$ in radius direction can be expressed as equation (2), the irradiated power $P$ should be determined by integration of equation (3).

$$
\begin{aligned}
& I(r)=I_{0} \cdot \exp \left(-a \frac{r^{2}}{r_{0}^{2}}\right) \\
& \Delta P=\Delta r \times I(r) \times 2 \pi r
\end{aligned}
$$

Table 1 Basic specification of laser oscillator.

\begin{tabular}{l|c}
\hline \multicolumn{1}{c|}{ Item } & Value \\
\hline \hline Type & IB Laser DiNY $\mathrm{pQ}$ \\
\hline Wavelength & $355 \mathrm{~nm}$ \\
\hline Pulse modulator & EO-Q switch \\
\hline Beam Mode & $\fallingdotseq$ TEM00 \\
\hline Pulse duration & $\leqq 10 \mathrm{~ns}$ \\
\hline Repetition rate & $\leqq 1 \mathrm{kHz}$ \\
\hline Average power & $\leqq 1.2 \mathrm{~W}$ \\
\hline Pulse energy & $\leqq 1.3 \mathrm{~mJ}$ \\
\hline Beam diameter & $1.5 \mathrm{~mm}$ \\
\hline Divergence & $1.1 \mathrm{mrad}$ \\
\hline
\end{tabular}

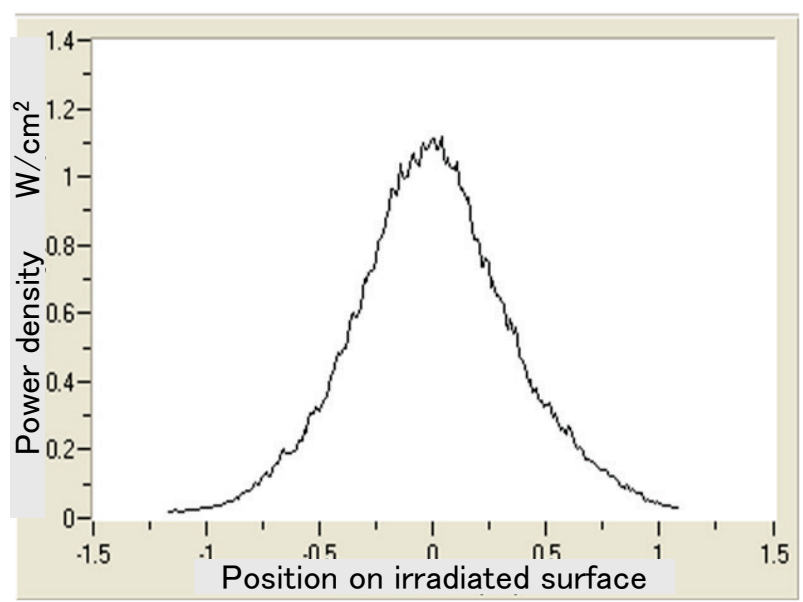

Fig. 1 Typical beam profile of pulsed YAG laser.

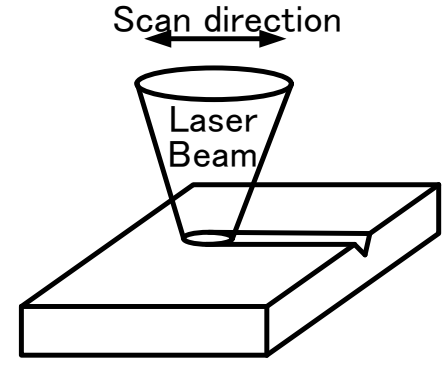

a) Groove generation.

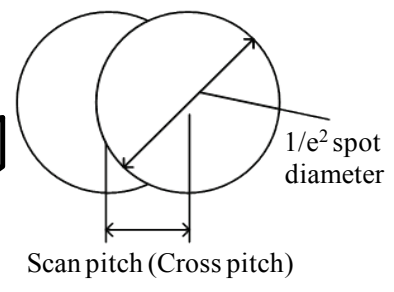

(b) Overlap of pulses.
Fig. 2 Irradiation condition for groove machining.

Table 2 Irradiation conditions for experiments.

\begin{tabular}{l|c}
\hline \multicolumn{1}{c|}{ Item } & Value \\
\hline \hline Workpiece material & $\mathrm{Si}, \mathrm{Al}$ and $\mathrm{Ag}$ \\
\hline Fluence & $0.4 \sim 2.5 \mathrm{~J} / \mathrm{cm}^{2}$ \\
\hline Overlap rate & $1 / 2$ of spot diameter \\
\hline Pass number & 40 times \\
\hline Focal length & 50,100 and $140 \mathrm{~mm}$ \\
\hline $1 / \mathrm{e}^{2}$ Spot diameter & 4,8 and $11 \mu \mathrm{m}$ \\
\hline
\end{tabular}




$$
P=\int d P=\int_{0}^{\infty} 2 \pi r \cdot I(r) \cdot d r
$$

On the other hand, $P$ is also related with pulse duration $\tau$ and pulse energy $E_{p}$ as expressed in equation (4). When the spot radius is set as the $1 / \mathrm{e}^{2}$ radius, the constant $a$ becomes 2 , and then, $I(r)$ can be expressed as equation (5) based on equations from (1) to (4).

$$
\begin{aligned}
& P=\frac{E_{p}}{\tau} \\
& I(r)=\frac{2 E_{p}}{\pi \cdot \tau \cdot r_{1 / e 2}{ }^{2}} \exp \left(-2 \frac{r^{2}}{r_{1 / e 2}^{2}}\right)
\end{aligned}
$$

Figure 4 (b) schematically indicates the model of convergence behavior caused by an objective lens. By assigning $\theta$ and $L$ as the angle of divergence of the laser source and the optical length from the entrance of the oscillator to the objective lens respectively, if $L$ is enough large, the beam diameter $W$ introduced into the objective lens can be expressed as equation (6) [16]. By converging the beam with the objective lens of the focal length $F$, the spot diameter $W$ becomes as expressed in equation (7) [16]. Since this relationship is assumed for the $1 / \mathrm{e}$ diameter, the $1 / \mathrm{e}^{2}$ radius $r_{1 / e^{2}}$ should be expressed as equation (8). By substituting $r_{1 / e^{2}}$ into the above mentioned equation (5), the intensity distribution $I(r)$ at the focal point can be derived as the function of radial position $r$, focal length $F$ and the laser irradiation parameters such as $E_{p}$ and $\tau$.

$$
\begin{aligned}
& W^{\prime}=\theta \times L \\
& W=\frac{f \cdot \lambda}{\pi \cdot W^{\prime}} \\
& r_{1 / e^{2}}=\frac{W}{\sqrt{2}}
\end{aligned}
$$

\subsection{Ablation model considering intensity distribution}

Figure 5 shows the experimental results of silicone grooving by using the objective lens of focal length 140 $\mathrm{mm}$. From the experiments, the relationships between the machined width and the intensity distributions are obtained as plotted in this figure. Here, fluence of irradiated laser are changed within range from 0.5 to $5 \mathrm{~J} / \mathrm{cm}^{2}$ by using attenuator. As shown in the figure, power densities at the edge of the machined widths are almost constant regardless of the irradiated fluence. Therefore, threshold power density $P_{t h}$ can be determined as a mean value of those power densities. For instance, $P_{t h}$ of silicone is $78 \mathrm{MW} / \mathrm{cm}^{2}$.

On the other hand, Fig. 6 shows the change in machined width according to the change in the average power density in the same experiments. The decrease in power density makes the machined width become small gradually. Conventionally, the threshold is often determined as the power density $P_{t h}$ that becomes the X intercept or the fluence corresponding to $P_{t h}$. However, the changing behavior of the machined width changes corresponding to the spot diameter of the irradiated laser. This means that the conventional definition of threshold is affected with configuration of the

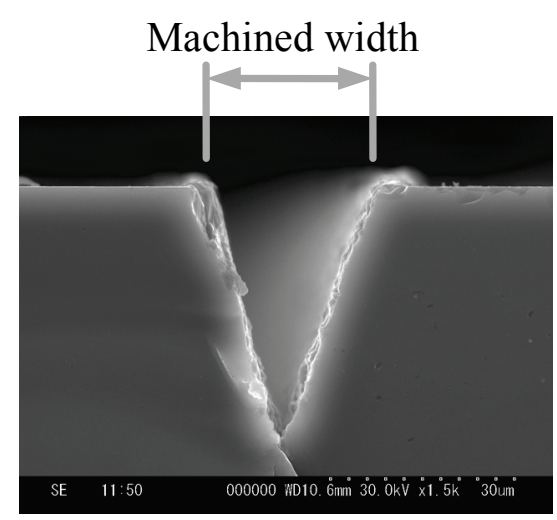

Fig. 3 Definition of width of machined groove.

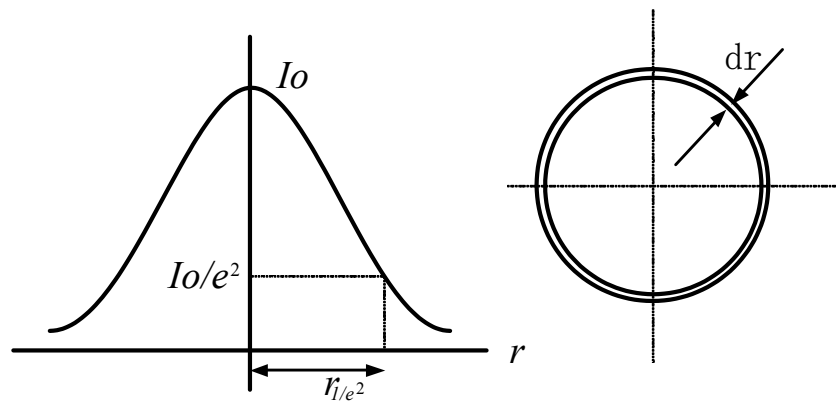

(a) Intensity distribution of TEM00 mode laser.

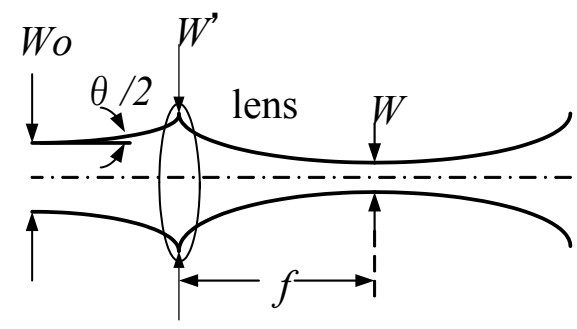

(b) Model of convergence by an objective lens.

Fig. 4 Model of laser intensity distribution at focal point.

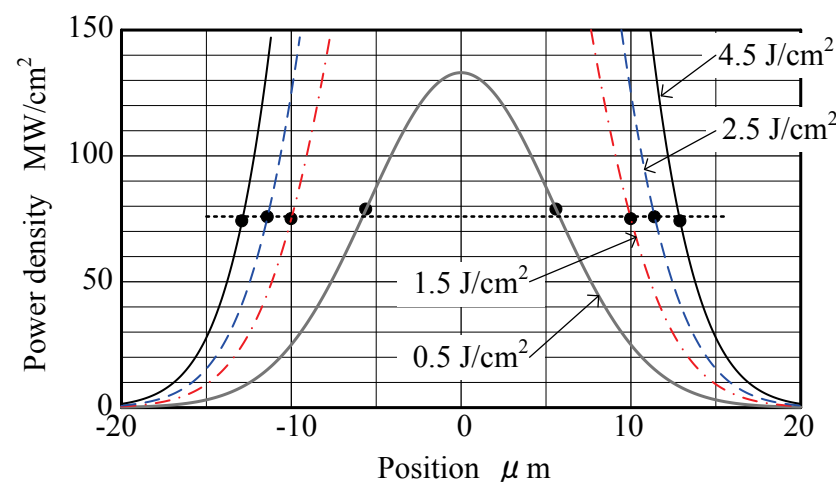

Fig. 5 Relationship between groove width and intensity distribution for Si grooving.

(Focal length $=140 \mathrm{~mm}$ ) 
optical system, and this disturbs the rational determination of the ablation conditions.

Figure 7 schematically indicates the relationship between the machined width and the intensity distribution. In this figure, the machined depth and the spot diameter are shown as $d$ and $d_{1 / e^{2}}$ respectively. As shown in Fig.6, the machined widths intersect with intensity distribution curves at constant power density $P_{t h}$. Therefore, in case of the intensity distribution of dashed line, since the maximum intensity corresponds to $P_{t h}$, the machined width should be 0 , consequently, this intensity corresponds to the $\mathrm{X}$ intercept in Fig. 6 .

At this time, the average power density $P_{\text {tho }}$ for this intensity curve is derived from the sum total of intensity divided with spot diameter $d_{1 / e^{2}}$. However, in case of the intensity distribution represented by solid line, the intensity region within the range from $P_{t h 0}$ to $P_{t h}$ cannot remove a material. This means that $P_{t h 0}$ is not suitable for the ablation threshold. Furthermore, since $d_{1 / e^{2}}$ changes corresponding to the focal length of the objective lens, $P_{t h 0}$ is also changed. This indicates that the conventionally defined threshold $P_{t h 0}$ changes in the intensity distribution and the optical s stem configuration even if the material is same. The essential cause of this confliction is that the conventional definition does not consider the effect of the intensity distribution.

To overcome this problem, the novel definition of the ablation threshold is proposed by considering the intensity distribution at the focal point. As experimentally confirmed in Fig.6, the machined width intersects with the intensity distribution curve at the same power density $P_{t h}$ independent of fluence of the irradiated laser. This indicates that the laser intensity exceeding $P_{t h}$ can remove the material selectively in the irradiated workpiece surface. Therefore the value of $P_{t h}$ is proper for the ablation threshold. In next section, the validity of the definition will be confirmed experimentally.

\section{Threshold of various materials and its application}

\subsection{Determination of threshold of silicone}

Figure 8 shows the experimental results of silicone grooving by using the objective lenses of focal length 100 and $50 \mathrm{~mm}$. The figure of (a) shows the results obtained by using the lens of focal length $f=100 \mathrm{~mm}$. Regardless of fluence, the intersection power density $P_{t h}$ between the machined width and the intensity distribution curve are constant, and its values are almost $75 \mathrm{MW} / \mathrm{cm}^{2}$ similar to Fig.6. Furthermore, as shown in plots of (b), even if $f$ is reduced to $50 \mathrm{~mm}, P_{t h}$ is independent of fluence, and its values are also almost $75 \mathrm{MW} / \mathrm{cm}^{2}$ constantly.

Table 3 shows the values of spot diameter, machined width and threshold corresponding to the focal length. The machined width is measured of the common fluence of $4 \mathrm{~J} / \mathrm{cm}^{2}$. The threshold values are compared between the conventional and proposed definition. The spot diameter and machined width is decreasing with decrement in focal length. Therefore, in case of the conventional definition, the values of threshold become small according to decreasing the spot diameter. On the other hand, in case of the novel definition, the values of threshold could keep

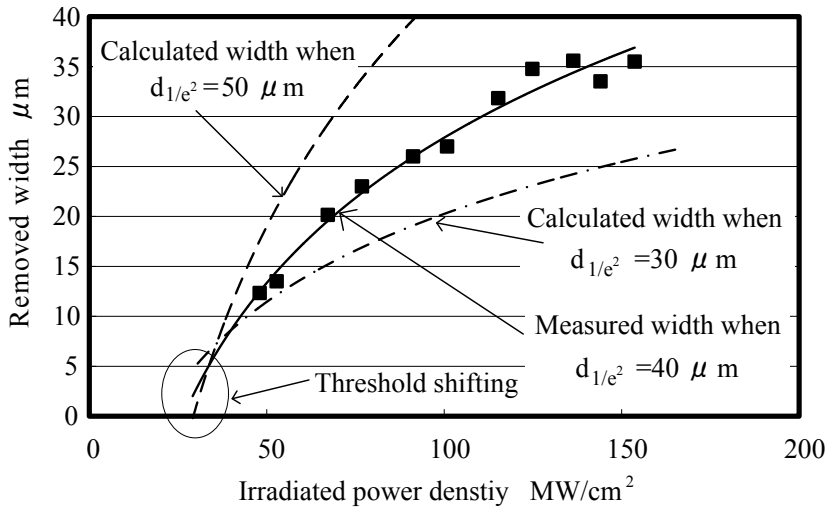

Fig. 6 Change in groove width with average power density.

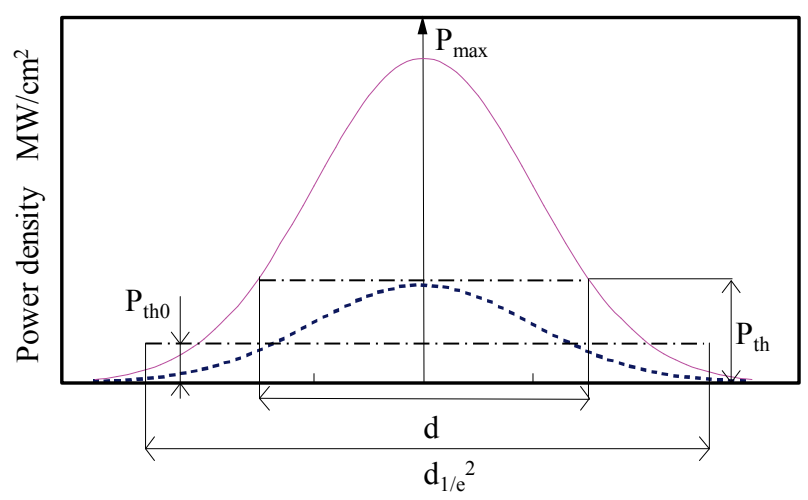

Fig. 7 Definition of ablation threshold.

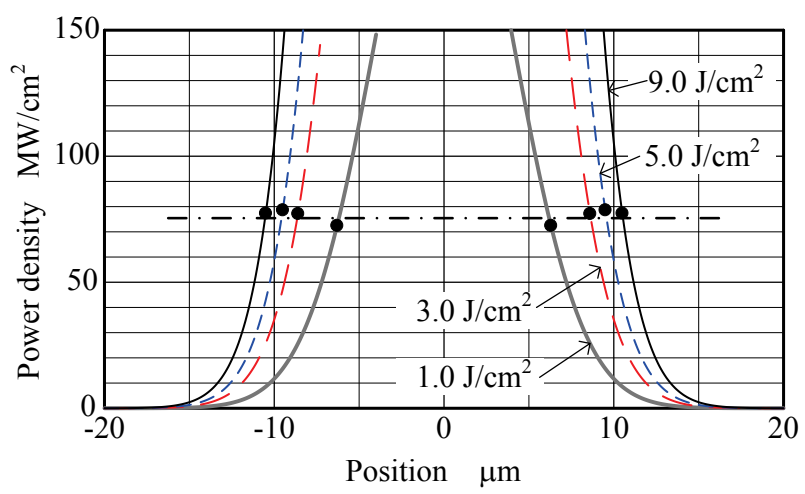

(a) Focal length $=100 \mathrm{~mm}$

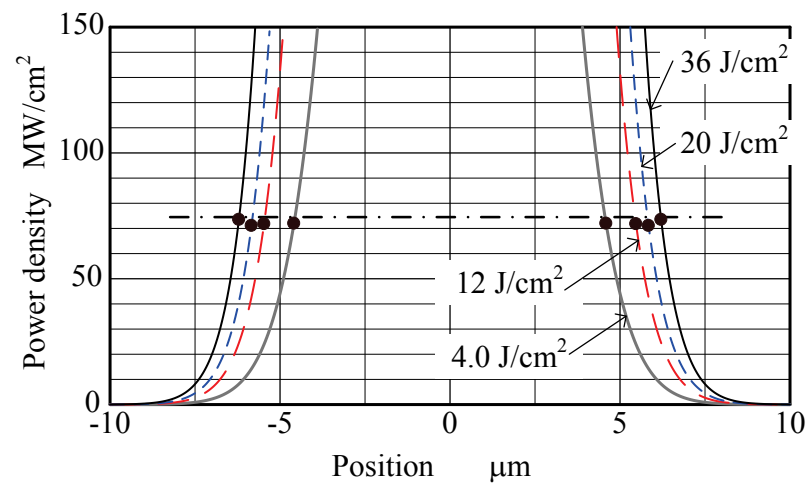

(b) Focal length $=50 \mathrm{~mm}$

Fig. 8 Relationship between groove width and intensity distribution for Si grooving. 
constant because the definition considers the effect of the intensity distribution.

\subsection{Determination of threshold for other materials}

Figure 9 shows the measured results of the threshold in case of aluminum ablation. Plot of (a) and (b) indicate the relationships between the machined width and the intensity distribution curve for focal length $F$ of 140 and 50 $\mathrm{mm}$ respectively. In case of aluminum, regardless of fluence, the intensities at intersection between the machined width and the intensity distribution curve are constant similar to silicone, but the value of threshold is almost 135 $\mathrm{MW} / \mathrm{cm}^{2}$ that is different to the value of silicone. In addition, the threshold values are also constant independent of the focal length.

On the other hand, Fig.10 shows the determination of the threshold for silver ablation. In case of silver, the value of threshold is $40 \mathrm{MW} / \mathrm{cm}^{2}$. Similar to other materials, the threshold of silver is also constant independent of fluence of irradiated laser.

From these results, it is found out that the ablation threshold based on the proposed definition is determined constant independent of laser irradiation condition and optical system configuration. This indicates that the threshold can be determined as the inherent value for materials.

\subsection{Application of threshold determined by proposed definition}

As shown in Fig.5, the machined width changes with the change in fluence of irradiated laser. However, since the width depends upon threshold and the intensity distribution, the width can be estimated from the relationship of both.

Equation (9) indicates the relationship between threshold power intensity and the machined width $W_{m}$. Thus, $W_{m}$ can be estimated from this relationship. For the estimation, $W_{m}$ can be transposed to left side, and then, equation (10) is derived. $W_{m}$ can be estimated from ablation threshold $P_{t h}$, pulse energy $E_{p}$, pulse duration $\tau$ and spot radius $r_{1 / e^{2}}$. Furthermore, since spot radius depends upon divergence angle $\theta$ and focal length $F$, by considering those parameters, the effects of not only the laser irradiation conditions but also the optical system configuration can be taken in account. This relationship is especially effective to ablation with weak laser irradiation that slightly exceeds threshold. At this time, those lowpowered ablation suppresses the generation of the heat affected zone (HAZ), and enables to machine small holes and grooves that are smaller diameter and width than the spot diameter respectively.

$$
\begin{gathered}
P_{t h}=I_{\left(W_{m}\right)}=\frac{2 E_{p}}{\pi r_{1 / e^{2}}^{2} \cdot \tau} \exp \left(-2 \frac{W_{m}^{2}}{r_{1 / e^{2}}^{2}}\right) \\
W_{m}=\sqrt{2 \cdot r_{1 / e^{2}}^{2} \cdot \ln \left(\frac{2 E_{p}}{P_{t h} \cdot \pi r^{2} \cdot \tau}\right)}
\end{gathered}
$$

Table 3 Change in spot diameter, machined width and threshold corresponding to focal length.

\begin{tabular}{l|c|c|c}
\hline \multicolumn{1}{c|}{ Focal length } & $\mathrm{F}=50 \mathrm{~mm}$ & $\mathrm{~F}=100 \mathrm{~mm}$ & $\mathrm{~F}=140 \mathrm{~mm}$ \\
\hline \hline Spot diameter & $8.02 \mu \mathrm{m}$ & $16.3 \mu \mathrm{m}$ & $21.9 \mu \mathrm{m}$ \\
\hline $\begin{array}{c}\text { Machined width } \\
\left(4 \mathrm{~J} / \mathrm{cm}^{2}\right)\end{array}$ & $9.0 \mu \mathrm{m}$ & $18.2 \mu \mathrm{m}$ & $25.0 \mu \mathrm{m}$ \\
\hline $\begin{array}{l}\text { Thershold value } \\
\begin{array}{l}\text { Thershold value } \\
\text { (Conventional) }\end{array}\end{array}$ & $74.4 \mathrm{MW} / \mathrm{cm}^{2}$ & $75.3 \mathrm{MW} / \mathrm{cm}^{2}$ & $77.0 \mathrm{MW} / \mathrm{cm}^{2}$ \\
\hline
\end{tabular}

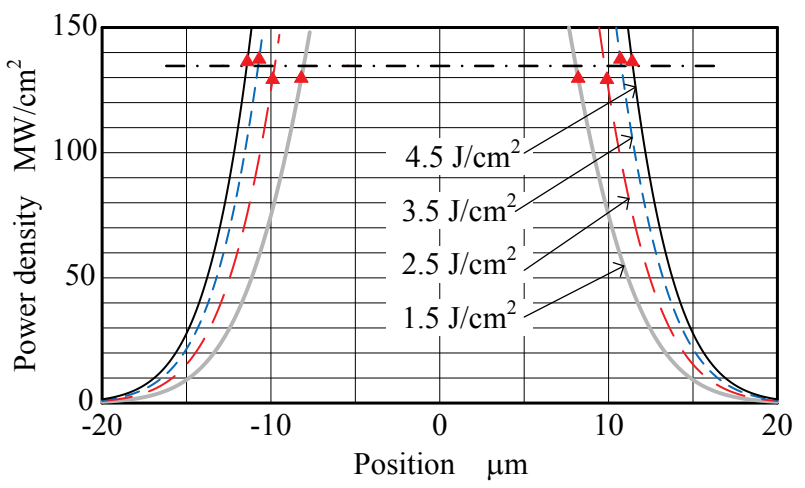

(a) Focal length $=140 \mathrm{~mm}$

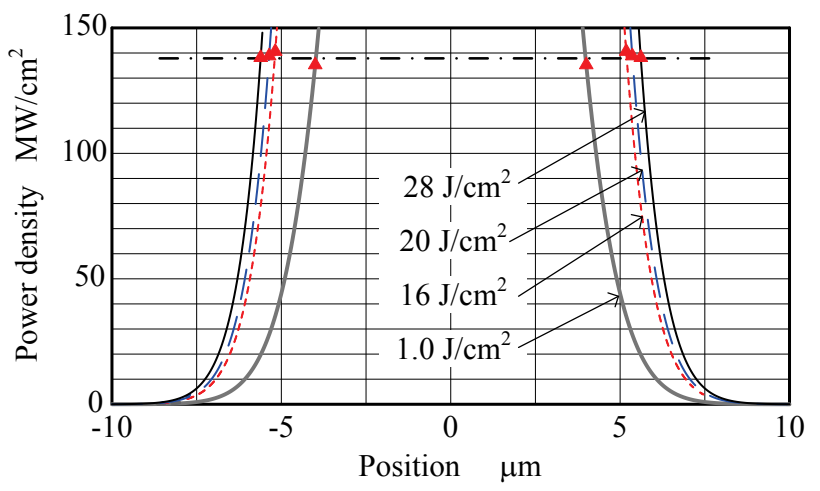

(b) Focal length $=50 \mathrm{~mm}$

Fig. 9 Relationship between groove width and intensity distribution for $\mathrm{Al}$ grooving.

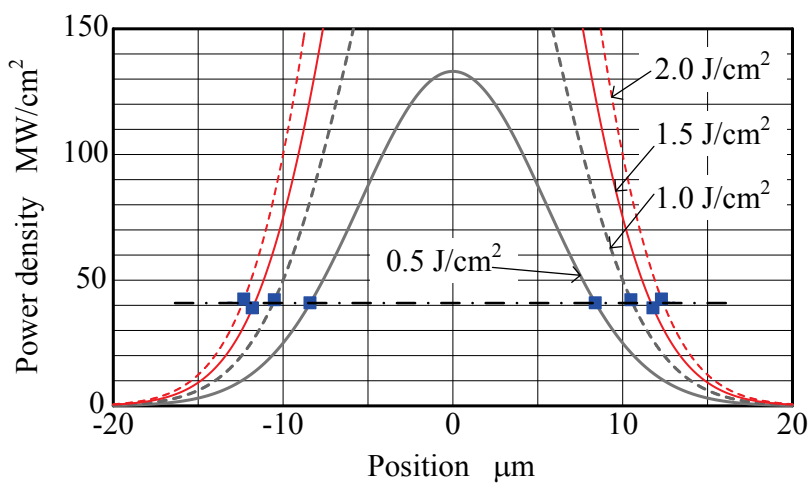

Fig. 10 Relationship between groove width and intensity distribution for Ag grooving.

(Focal length $=140 \mathrm{~mm}$ ) 


\section{Conclusion}

The novel definition of ablation threshold considering the intensity distribution of the irradiated laser is proposed, and then, its validity is examined experimentally. From the results, following are made clear.

(1) The machined width and the intensity distribution curve, which is derived from the converging characteristics of laser, intersect at constant power density independent of fluence. Therefore, the power density at intersection can be newly defined as the ablation threshold

(2) The proposed threshold is determined as the constant value independent of the focal length of the objective lens.

(3) The values of threshold are different in materials. Those of silicone, aluminum and silver have been determined for nano-second pulsed YAG laser as 80,135 and $40 \mathrm{MW} / \mathrm{cm}^{2}$ respectively.

\section{References}

[1] S. K. Ray, K. Seshan, M. Interrante: Proc. of 40yh Electronic Components and Technology Conference, Las Vegas, (1990) p.395.

[2] H. Tabata, O. Murata, T. Kawai and S. Kawai: Appl. Phys. Lett., 56 (1990) 1576.

[3] T. Sasaki, H. Usui and N. Koshizaki: J. of Japan Laser Processing Soc., 14 (2007) 191.

[4] S. Wada, H. Tashiro, K. Toyoda, H. Niino and A. Yabe: Appl. Phys. Lett., 63 (1993) 211.

[5] H. C. Kapteyn, O. Cohen, Z. Xiaoshi, A. Lytle and M. M. Murnane: Laser Focus World, 43, 5 (2005) p.26.
[6] V. C. Coffey: Laser Focus World, 46, 5 (2008) p.26.

[7] T. Arai: "Beginner's book of laser processing" (Kogyo Chousakai Publ., Tokyo, 2004) p.174.

[8] S. Nishio: "Handbook of application to laser processing" ed. by H. Masuhara (NGT, Tokyo, 2006) p.46.

[9] Y. Ito: "Laser ablation and its applications" ed. by Inst. of Elec. Eng. of Japan (Corona Publ., Tokyo, 1999) p. 161 .

[10] R. Tatsumi: "Handbook of laser processing technology" ed. by m. Ikeda (Asakura Publ., Tokyo, 1992) p.421.

[11]I.Vladoiu, M. Stafe, I.M. Popescu: The Influence of Spot Diameter, Fluence and Wavelength of the Nanosecond Laser Pulses on the Ablation Rate of Aluminum, U.P.B. Sci. Bull., Series A, 69, 3 (2007) p.81.

[12] M. S. Komlenok, V. V. Kononenko, I. I. Vlasov, V. G. Ralchenko, N. R. Arutyunyan, E. D. Obraztsova, and V. I. Konov: Laser "Nano"ablation of Ultrananocrystalline Diamond Films, J. of Nanoelectronics and Optoelectronics, 4 (2009) p.286.

[13]N. Bityurin and A. Malyshev: Bulk photothermal model for laser ablation of polymers by nanosecond and subpicosecond pulses, J. of Applied Physics, 92, 1(2002) p.605

[14] S. Lazare and V. Grainier: Ultraviolet Laser hotoablation of Pplymers: A Review and Recent Results, Laser Chem., 10 (1989) p.25.

[15] P. Mannion, J. Magee, E. Coyne, G. M. Oconnor: Ablation thresholds in ultrafast laser micro-machining of common materials in air, Prec. Of SPIE, 4876 (2003) p.470.

[16] Y. Asami: "Laser Engineering” (Tokyo Denki University Press., Tokyo, 1972) p.27\&53.

(Received: July 06, 2011, Accepted: December 11, 2012) 